\title{
Compact Steep Spectrum and GHz-Peaked Spectrum radio sources
}

Carlo Stanghellini*

INAF Istituto di Radioastronomia

E-mail: Cstaneira.inaf.it

Compact Steep Spectrum (CSS) and GHz-Peaked Spectrum (GPS) radio sources are powerful yet compact radio sources whose spectra are generally simple and convex with a peak between 100 $\mathrm{MHz}$ and a few GHz. The study of these objects is a key element to understand the evolution of the powerful extragalactic FRI and FRII radio sources. There is a general consensus that they are small because they are young, but there are still open questions about their evolution, their interaction with the host galaxy interstellar medium, the physics of the jets and so on. I will review what we know about the CSS and GPS radio sources, and what we need to do to improve our understanding on their nature and evolution, with an emphasis on what we can learn by means of new observations in the radio domain.

8th European VLBI Network Symposium

September 26-29, 2006

Toruń, Poland

${ }^{*}$ Speaker. 

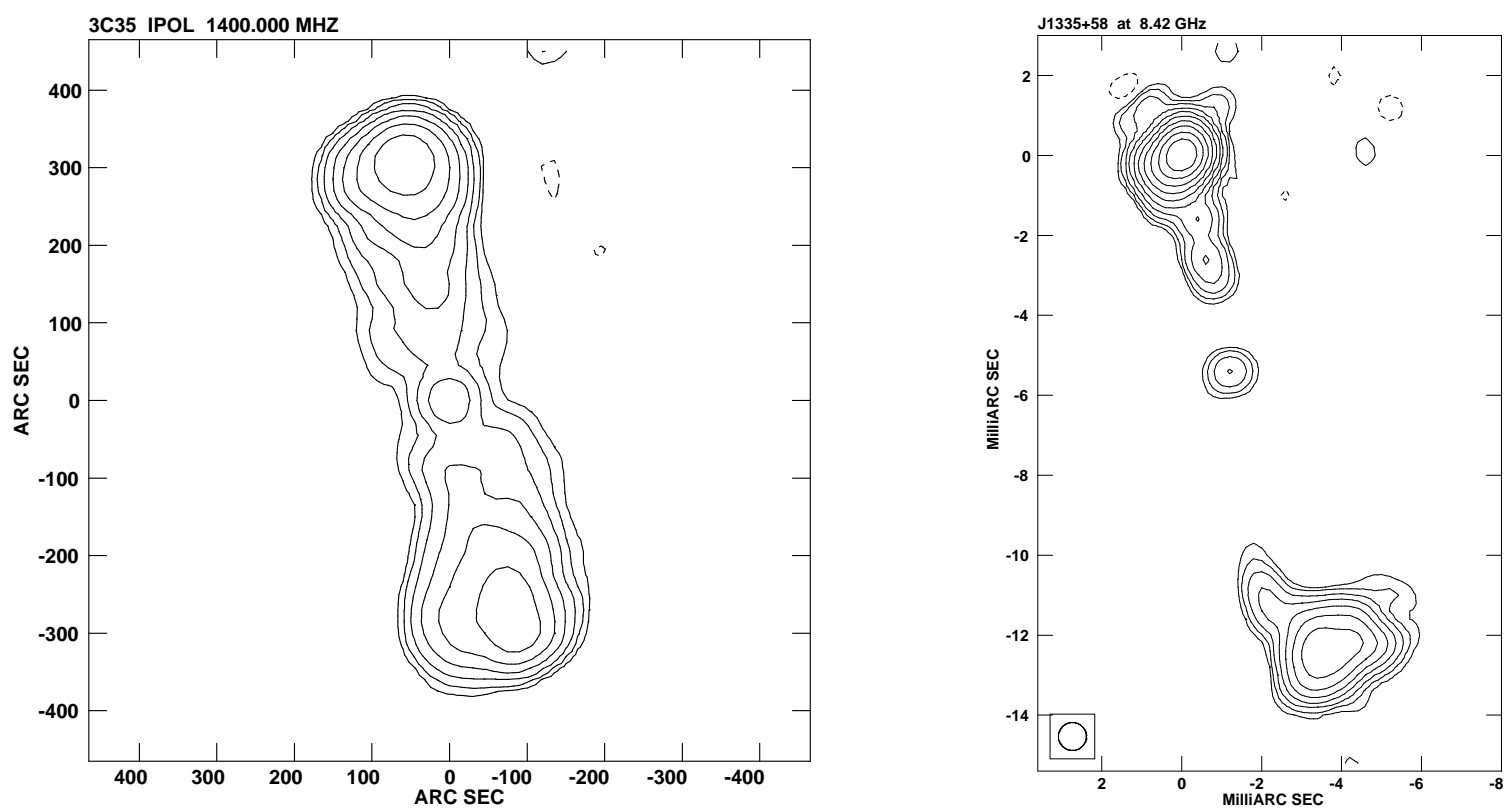

Figure 1: $3 \mathrm{C} 35$ (left) and $\mathrm{J} 1335+5844$ (right) have the same morphology on very different scales; $\mathrm{J} 1335+5844$ has an angular size of 14 milliarcseconds while $3 \mathrm{C} 35$ is $>50000$ times larger.

\section{Looking for young extragalactic radio sources}

Extragalactic radio sources originating from the centre of active galaxies grow to very large sizes, larger than the hosting galaxy itself, reaching sizes of hundreds of kpc and sometimes exceeding a Mpc over ages up to $10^{8}$ years, which is a relatively short time-scale compared to the age of the Universe. They are very relevant objects in the sky at radio wavelengths. To study and understand their origin and early stages of their evolution, it is important to find the youngest between them. To this purpose, we need to search for the most compact sources showing the same morphology as the largest ones, which means the presence of a two-sided structure characterized by lobes dominating the radio emission with two bright hot-spots at the edges, provided the angular resolution is sufficient to disentangle their emission within the lobes. In general, the radio core accounts for a tiny fraction of the total flux density on all scales, and, due to limitations of dynamic range, sometimes is undetected in the smallest objects.

\section{Compact Symmetric Objects and Medium-Size Objects}

These sources have been extensively studied in the past years. Based on their morphology, they are named Compact Symmetric Objects (CSO) when smaller than $\sim 1 \mathrm{kpc}$ and Medium-Size Objects (MSO) if they have sizes between $\sim 1$ and $\sim 20 \mathrm{kpc}$.

Complete samples of CSOs and MSOs are difficult (or practically impossible) to obtain because we would need to know the parsec-scale structure of all the compact sources above a given flux-density limit. Generally, CSOs and MSOs have convex radio spectra peaking around $1 \mathrm{GHz}$ and around $100 \mathrm{MHz}$, respectively. 

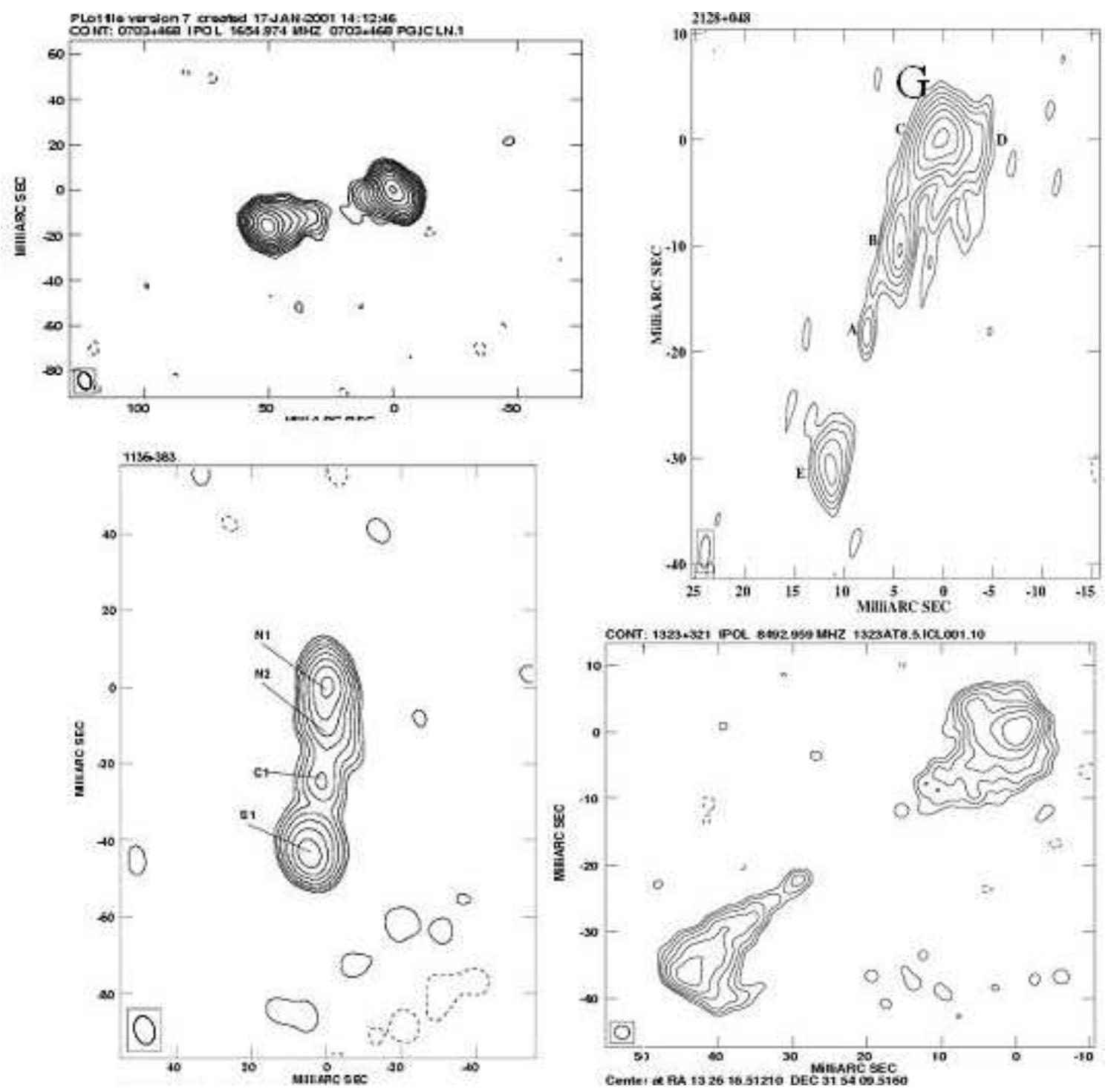

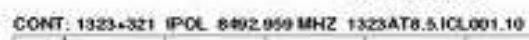

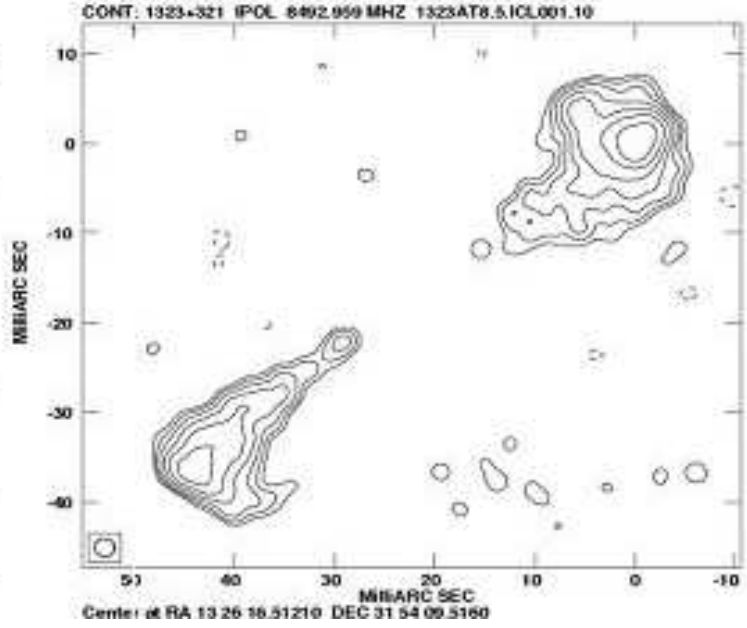

Figure 2: Other common morphologies of CSOs.

If the classification is based on the spectral shape, these sources are termed GHz-Peaked Spectrum (GPS) or Compact Steep Spectrum (CSS) radio sources. As expected for radio sources emitting synchrotron radiation, there is a strong anti-correlation between size and peak frequency (O'Dea 1998). In an attempt to select the youngest sources, Dallacasa et al. (2000) introduced the class of High-Frequency Peakers (HFP), including the more extreme GPS whose spectral peaks are above $5 \mathrm{GHz}$, which should be the smallest and so the youngest sources, if small means young. But does small really mean young?

\section{Young or frustrated?}

In the past years, two main scenarios have been proposed to explain the compactness of GPS/CSS radio sources. 


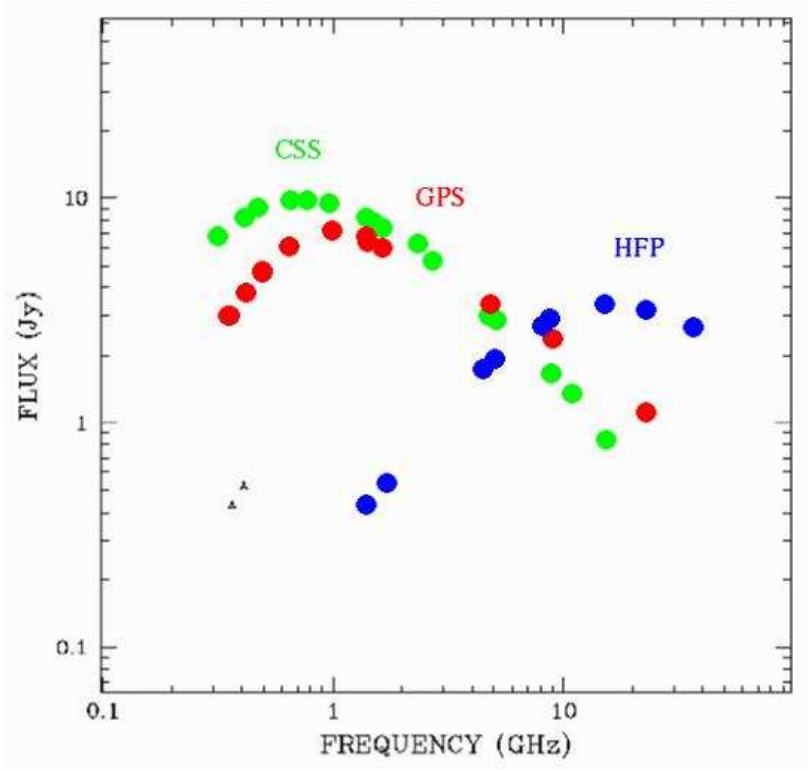

Figure 3: Typical radio spectra of CSS, GPS, and HFP sources.

The youth scenario (Phillips and Mutel 1982, Fanti et al. 1995, Readhead et al. 1996, O'Dea \& Baum 1997; Alexander 2000; Snellen et al. 2000, 2003), which is the natural explanation for small sources evolving into large ones, suggests that GPS sources with symmetric morphology are small because they are young, and that in the course of their life they will expand outwards in a self-similar way.

In the frustration scenario (e.g., van Breugel et al. 1984; De Young 1993; Carvalho 1994, 1998), the radio emitting plasma is confined (for the lifetime of the radio emission) to a region within the host galaxy by an external medium that is dense enough to prevent the expansion of the radio source.

Both the "youth" and "frustration" scenarios are motivated by the morphological similarity between the small GPS/CSS galaxies and the extended radio galaxies, while the frustration scenario, if valid for most GPS/CSS sources, can also explain the evidence that there are too many small sources if they spend at constant luminosity only $1 / 100$ or $1 / 1000$ of their lives in the GPS/CSS phase.

Variations or complications of these scenarios may include recurrency, which means radio sources die and start again several times, and/or the possibility that a fraction of them are short lived, i.e. can die young because of episodic fuelling, maybe triggered by a recent merger event.

To see whether there is enough gas to confine and stop the expansion of the radio emitting plasmoids, we can try to probe the central regions of the hosting galaxies by means of radio observations at $21 \mathrm{~cm}$ to look for HI (Pihlström et al. 2003, Vermeulen et al. 2003, Orienti et al. 2006), infrared observations to find cold gas (Fanti et al. 2000), X-ray observations to find X-ray emitting hot gas or absorbing cold gas (Guainazzi et al. 2006, Vink et al. 2006) or we can study 

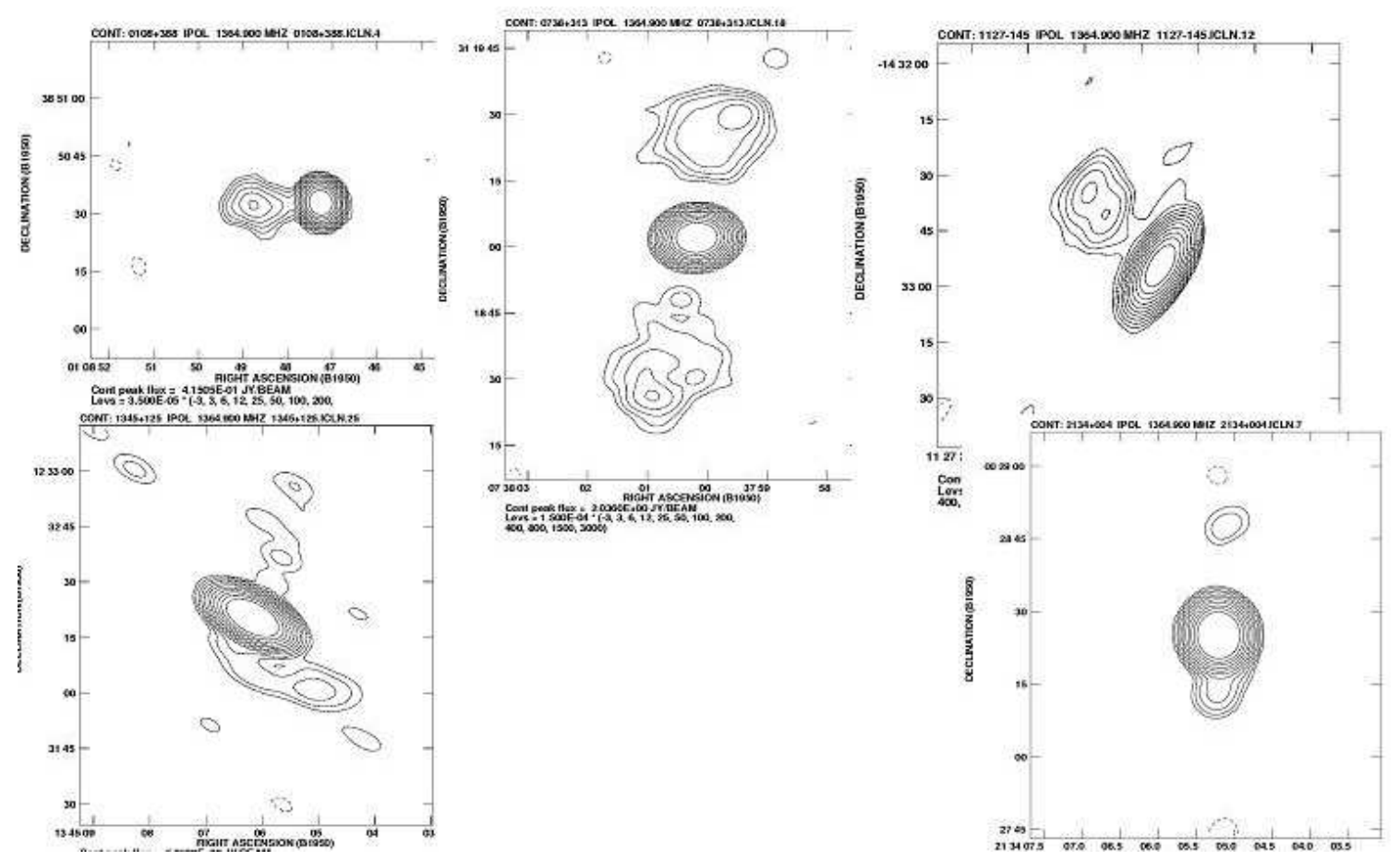

Figure 4: Examples of extended emission around GPS radio sources.

the depolarization of the radio emission to look for ionized gas (Fanti et al. 2004). Even if substantial information can be derived from these observations, there is no conclusive evidence of the presence of enough gas to stop the expansion. On the other hand, on the very small scale $(<1 \mathrm{kpc})$, it seems that the gas, when present in great quantity, is confined to a disk and/or torus in a plane perpendicular to the radio axis, and therefore not directly interacting with the expanding jets.

A strong support for the youth scenario comes from the determination of the age by means of the direct observation of the radio source expansion. In GPS radio sources with well-defined hotspots, observed over time-baselines spanning several years, relative separation speeds of $0.1-0.3 c$ have been found, implying ages of the order of a few thousand years (see the review by Polatidis and Conway 2003). The evidence for youth is strengthened by the determination of the age derived by the steepening of the radio spectrum caused by the radiative losses. In the lobes of GPS, where the old electrons are deposited during the lifetime of radio sources, ages of thousands of years have been calculated (Murgia 2003), well in agreement with the dynamical ages determined by expansion velocities.

\section{Extended emission}

A potential problem of the youth scenario comes with the detection of extended emission around some GPS/CSS radio sources. As an example, VLA observations (Fig. 4) show the GPS radio source (the unresolved compact component) surrounded by a faint emission on the arcsecond scale. Even if this emission does not contribute much to the total radio spectrum (unless going to very low frequencies), it has strong implications for the nature of these GPS radio sources. Radio 

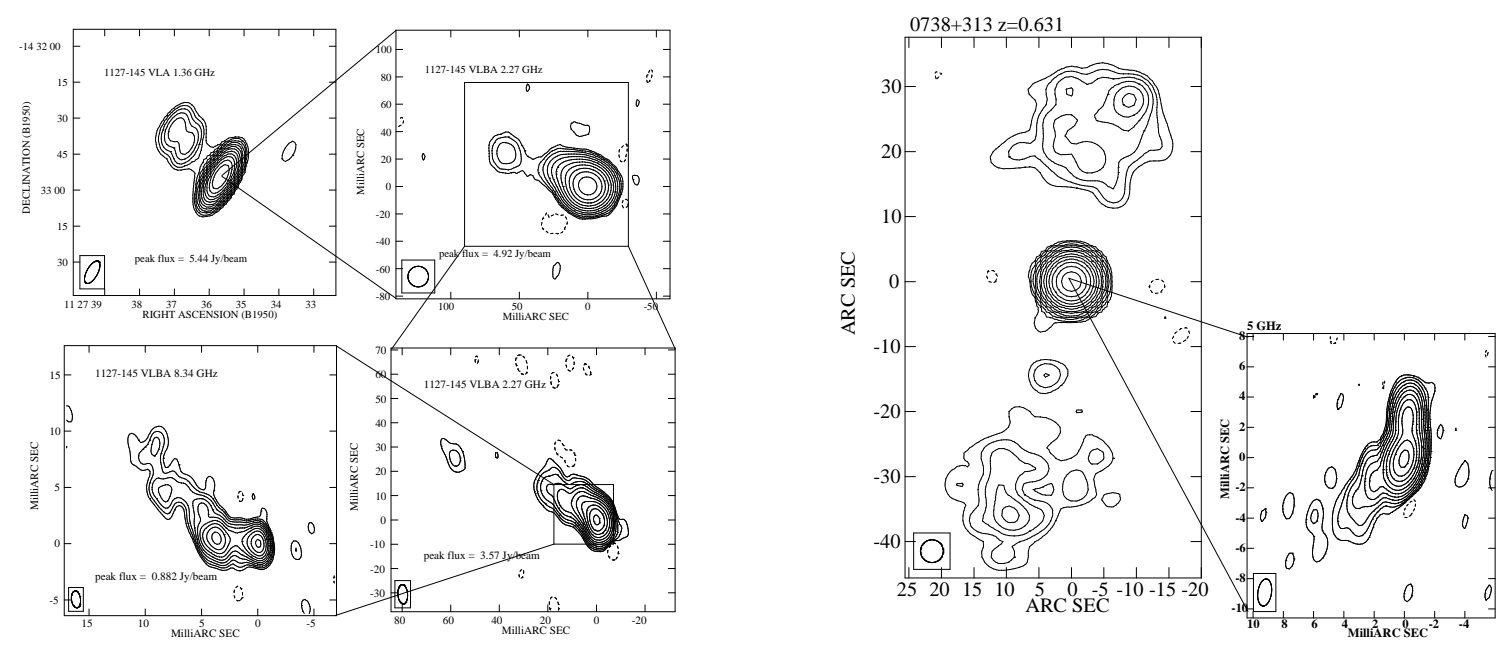

Figure 5: From parsec to kpc scale: B1127-145 (left) and B0738+313 (right)

emitting regions extending for tens of kpc cannot form in thousands of years but rather in millions of years, in strong contrast with the youth scenario.

A popular explanation to interpret the extended emission and save the youth scenario is to assume recurrency in the radio activity (Baum et al. 1990). If the radio activity is a recurrent phenomenon during the life of an active galaxy, we can see the relic emission of a past activity not yet completely vanished due to radiative and expansion losses, while a new radio source is forming and growing, provided the duty cycle is shorter than $10^{7}-10^{8}$ years.

Recurrency is not always the answer to explain extended emission and alternative (and sometimes more likely) explanations should be worked out. In several sources we may be witnessing ongoing (or at least recent) transport of radio plasma from the core to the extended structure.

In Fig. 5, we see two examples of GPS radio sources, where recurrency has seriously been taken into consideration to explain the presence of the extended emission with little or no justification for that.

In the case of the GPS quasar $0738+313$ with a core-jet mas morphology, we see the hotspots on arcsecond scales, which suggest that there is a continuous supply of fresh electrons from the nucleus. In the quasar 1127-145, which also has a core-jet mas morphology, we see radio emission from the mas to the arcsecond scale. Again, this suggests the existence of a continuous flow of relativistic electrons from the core to the arcsecond-scale jet, which rules out the recurrency hypothesis and contradicts either the youth or the frustration scenario. A key fact is that these two sources are quasars and this will be discussed in the next section.

\section{The nature of GPS quasars}

The relationship between GPS galaxies and quasars has been a longstanding question. Are the GPS quasars either the beamed counterparts of the GPS radio galaxies as predicted in Unified Scheme Models (e.g. Urry \& Padovani 1995) or are they unrelated objects simply having similar radio spectra? The idea that the GPS quasars and radio galaxies are different classes of object has been discussed by Snellen (1997) and Stanghellini et al. (2001). 
A plausible hypothesis is that GPS quasars exhibiting a core-jet or complex morphology on the mas scale are indeed "extended" (namely with lobes larger than tens/hundreds of $\mathrm{kpc}$ ), coredominated radio sources, whose redshifts are sufficiently high that most (if not all) of the large-scale structure is below our current detection threshold, and when extended emission is eventually detected it can easily be explained in terms of an active radio source currently supplied with fresh electrons by the jets coming from the active nucleus. This would imply that GPS quasars with core-jet mas morphology represent an intermediate population of objects, less beamed, but morphologically similar to the common flat-spectrum radio sources that dominate the radio surveys at cm wavelengths.

We can also note that quasars are not present in the same fraction in different samples of GPS and CSS. The incidence of quasars and/or core-jet morphologies is much higher in the samples of the smaller and brighter objects (Stanghellini et al. 2005).

GPS spectra in quasars and galaxies thus originate from intrinsically different emitting regions: micro lobes/hotspots with a large range of sizes in galaxies, while in the quasars the emitting region tends to be more compact and closer to the core. The structure in the GPS quasars may also be simpler with most of the emission coming from one or a few knots in a moderately beamed jet. These knots may correspond to the location of a shock front, where the electrons are re-accelerated or a turn in a helical jet, where the bulk flow is locally pointing toward the observer, enhancing its brightness by moderate relativistic beaming.

An important conclusion is that quasars are contaminating objects and should be excluded from any discussion regarding the evolution of extragalactic radio sources. To this purpose, the best approach is to consider only HFP/GPS/CSS sources with a two sided (VLBI) morphology. This is difficult to accomplish because the selection of samples often lacks such information, being mostly based on the shape of the radio spectrum. As a first step, we can therefore consider only the HFP/GPS/CSS radio sources associated to galaxies, possibly with the addition of a very few quasars known to have a genuine two-sided morphology.

Even if we get rid of the contaminating objects in our sample of HFP, GPS, and CSS radio sources, these objects are too numerous. The number counts can be in agreement with the youth scenario if there is a decrease in luminosity during the evolution (Fanti et al. 1995) and/or there is a fraction of them which die young, before growing up.

Therefore, in a qualitative view, radio sources born as HFP expand to GPS sources, then to CSS sources, and finally they become the extragalactic radio sources of high (FRII) or low (FRI) luminosity.

\section{Modelling the radio source growth}

First analytical model for the first stage of a radio source growth was presented by Begelmann (1996). In his model, the expansion velocity depends weakly on the source size, while the luminosity decreases with the square root of the size, giving rise to FR Is or FR IIs depending on the initial luminosity. Begelman does not compare the predictions of his model with the observations.

To have a quantitative and rigorous approach, we should compare models of a radio source evolution with the distributions of linear sizes of the samples of young radio sources. 
Several samples of HFP, GPS, and CSS sources have been selected in the past years (Fanti et al. 1990; Stanghellini et al. 1998; Snellen et al. 1998, Dallacasa et al. 2000, Bolton et al. 2004, Edwards and Tingay 2004, Snellen et al. 2002, Snellen et al. 2004, Spencer et al. 1989, Marecki et al. 1999, Fanti et al. 2001, Kunert et al. 2002, etc.). They cover different places of the turnover frequency - brightness domain, thus they are not simply duplication of each other.

Three evolutionary models have been developed in more recent years (Snellen et al. 2000; Alexander 2000; Tinti \& De Zotti 2006), which make use of several of the samples mentioned above. They have significant different predictions about self-similar expansion, radio-power evolution, existence of short-lived objects, cosmological evolution of the luminosity function, etc. These differences strongly depend on the assumptions made to combine the different samples, and on the corrections applied to account for the various selection criteria.

It is evident that the observational data are not sufficient to constrain the models, as there are too many different samples, each one covering only a fraction of the peak-frequency - brightness domain. Therefore, a single complete sample of a few hundreds objects spanning more than two decades in turnover frequency, and with a low flux-density limit would be extremely useful to better constrain the models. This can be obtained by means of selecting a completely new sample, or complementing/combining some of the existing complete samples with new observations to avoid the problems mentioned above.

Another crucial piece of information to constrain these evolutionary models is the measurement of the expansion velocity since it is related to the radio power and to the local ambient density. Reliable proper-motion measurements based on hot-spot velocity separation are available only for about a dozen sources, including upper limits, while for additional dozen sources with only sparse observations, proper motions are suspected (Polatidis \& Conway 2003, Gugliucci et al. 2005). Furthermore, the expansion speed of $0.1-0.3 c$ have been measured for the bright and luminous objects. It is possible that weaker radio sources have a slower growth or are even confined. It is, therefore, essential to increase the number of reliably measured expansion velocities in order to make a statistically significant analysis. This can be achieved thanks to multi-epoch VLBI monitoring of a large number of GPS and CSS with the currently available VLBI networks. The detection of proper motions in GPS and CSS requires high-resolution observations spanning several years, and it is therefore a time-consuming effort. However, it is a very powerful tool to understand the dynamical evolution of newly-born radio sources and set constraints on the current models. By increasing the number of sources with measured expansion (or with stringent limits), we can correlate it to the size, power and morphology of these sources and test the existence and fraction of frustrated objects.

\section{References}

[1] Alexander P. 2000, MNRAS 319, 8

[2] Baum S.A., O’Dea C.P., Murphy D.W., de Bruyn A.G. 1990, A\&A 232, 19

[3] Begelman M.C. 1996, Cygnus A - Studay of a Radio Galaxy, Proceedings of the Greenbank Workshop, held in Greenbank, West Virginia, 1-4 May, 1995. Edited by C.L. Carilli and D.E. Harris. ISBN 0521553431, Cambridge University Press, 1996., p.209

[4] Bolton R.C., Cotter G., Pooley G.G. et al. 2004, MNRAS 354, 485 
[5] van Breugel, W., Miley, G., Heckman, T., 1984, AJ, 89, 5

[6] Carvalho, J. C., 1994, A\&A, 292. 392

[7] Carvalho, J. C., 1998, A\&A, 329, 845

[8] Dallacasa, D., Stanghellini, C., Centonza, M., Fanti, R., 2000, A\&A, 363, 887

[9] De Young D.S 1993, ApJ 402, 95

[10] Edwards P.G. \& Tingay S.J. 2004, A\&A 424, 91

[11] Fanti R., Fanti C., Schilizzi R T. et al. 1990, A\&A 231, 333

[12] Fanti C., Fanti R., Dallacasa D., et al. 1995, A\&A, 302, 317

[13] Fanti C., Pozzi F., Dallacasa D., et al. 2001, A\&A, 369, 380

[14] Fanti C., Branchesi M., Cotton W.D. et al. 2004, A\&A, 427, 465

[15] Kunert M., Marecki A., Spencer R E. et al. 2002, A\&A 391, 47

[16] Gugliucci N.E., Taylor G.B., Peck A.B., Giroletti M. 2005, ApJ 622, 136

[17] Guainazzi M., Siemiginowska A., Stanghellini C. et al. 2006, A\&A, 446, 87

[18] Marecki A., Falcke H., Niezgoda J. et al. 1999, A\&AS 135, 273

[19] Murgia M. 2003 PASA 20, 19

[20] O’Dea, C.P., \& Baum S.A. 1997, AJ 113, 148

[21] O’Dea, C.P., 1998, PASP, 110, 493

[22] Orienti M., Morganti R., Dallacasa D. 2006, A\&A 457, 531

[23] Phillips, R.B., Mutel, R.L., 1982, A\&A, 106, 21

[24] Pihlström Y.M., Conway J.E., Vermeulen R.C. 2003, A\&A, 404, 871

[25] Polatidis A.G., Conway J.E. 2003, PASA 20, 69

[26] Readhead, A.C.S., Taylor, G.B., Xu, W., et al., 1996a, ApJ, 460, 612

[27] Snellen, I.A.G., 1997, PhD Thesis, Univ. of Leiden

[28] Snellen, I.A.G., Schilizzi, R.T., Miley, G.K., et al. 2000, MNRAS 319, 445

[29] Snellen I.A.G., Schilizzi R.T., de Bruyn A.G. et al.1998, A\&AS 131, 435

[30] Snellen I.A.G., Lehnert M.D., Bremer M.N. et al.2002, MNRAS 337, 981

[31] Snellen, I.A.G., Mack, K.-H., Schilizzi, R.T., Tschager, W., 2003, PASA, 20, 38

[32] Snellen I.A.G., Mack K.H., Schilizzi R.T., Tschager W. 2004, MNRAS 348, 227

[33] Spencer R E., McDowell J C., Charlesworth M. et al. 1989, MNRAS 240, 657

[34] Stanghellini, C., O’Dea, C.P., Dallacasa, D., et al., 1998, A\&AS, 131, 303

[35] Stanghellini C., Dallacasa D., O’Dea C.P. et al., 2001, A\&A 377, 377

[36] Stanghellini C., O’Dea C.P., Dallacasa D. et al. 2005, A\&A 443, 891

[37] Tinti, S.,, De Zotti, G. 2006, A\&A 445, 889

[38] Urry, C.M., Padovani, P. 1995, PASP, 107, 803

[39] Vermeulen R.C., Pihlström Y.M., Tschager W. et al.2003 A\&A 404, 861

[40] Vink J., Snellen I.A.G., Mack K.H., Schilizzi R.T. 2006, MNRAS 367, 928 